\title{
Autologous Fat Grafting Provides Good Outcomes as a Soft-Tissue Replacement in Hemifacial Atrophy
}

Jeon $\mathrm{FHK}^{1}$, Koneswaran $\mathrm{K}^{2}$, Varghese $\mathrm{J}^{1,2}$, Griffin $\mathrm{M}^{1,2}$, Frosdick $\mathrm{C}^{2}$, Butler $\mathrm{P}^{1,2}$

${ }^{1}$ Division of Surgery and Interventional Science, Uni versity College London, ${ }^{2}$ Department of Plastic Surgery, Royal Free Hospital, London

\section{Dear Editor,}

Progressive hemifacial atrophy, also known as Parry-Romberg syndrome (PRS), is a rare disease characterised by atrophy of the skin, subcutaneous tissues, cartilage and mimetic muscles of the hemiface. More severe cases lead to degeneration of the bony framework and can be accompanied neurologic and ophthalmologic complications, alopecia areata and changes in hair colour. The resultant asymmetry can have considerable psychological sequelae and treatment aims to reconstruct the soft tissue destruction and restore facial symmetry [1]. PRS is classically treated with microvascular free flaps as the gold standard, however this is associated with significant donor site morbidity, extended operative time, and risk of major complications such as flap failure. Furthermore there is a loss of sensation, poor skin colour match and multiple debulking procedures are required to improve the aesthetic outcome $[1,2]$. Autologous fat grafting is gaining prominence as the preferred method of soft tissue reconstruction. The soft nature of the graft results in a natural contour and improved skin quality, using a procedure that is safe, inexpensive and repeatable, making it the ideal filler [2]. However the successful reported use of fat transfer to treat hemifacial atrophy as a soft tissue filler are limited due to the concerns of effective fat graft survival.

To understand the efficacy of fat transfer in hemifacial atrophy we reflected on our experience. Data was collected from the electronic patient records to identify 19 patients treated with fat grafting for hemifacial atrophy between November 2006 May 2017. There was a with a female predominance (17 patients; $89 \%$ ) to males (2 patients; $11 \%$ ). Age of disease presentation was before the age of 10 in $60 \%$ (range 3-40 years old) and there was an equal distribution of disease affecting the right and left hemiface. Disease was in remission in $21 \%$ and active in $15 \%$ at time of surgery. Mean age at receiving fat transfer was at 37 years (range $17-66$ years). A mean volume of $18 \mathrm{ml}$ fat (range $4-67 \mathrm{ml}$ ) was grafted per procedure after preparation using the Coleman technique, and patients had a total of 2.68 procedures on average (range 1-8 procedures). Fat was predominantly harvested from the abdomen (58\%) and thighs (29\%), and were grafted most commonly to the cheek (25\%), temple (13\%) and chin (13\%) and nasolabial fold (11\%). Time between procedures ranged from 3.5 months to 6 years (median 8 months). Standardised patient reported outcome measures were not performed as part of routine care therefore clinic letters were examined for patient and surgeon reported outcomes. Clinic letters reported a high patient satisfaction at the outcome described as 'very pleased', 'very happy' and 'delighted'. Post-operative results were described by the surgeon as 'excellent', 'very well' and 'considerably improved' at 2 months -2 years follow-up (mean 11.65 months). Complications included one case of fat necrosis and 
2 cases of prominence on the grafted site. Figures $1 \mathrm{a}$ and $1 \mathrm{~b}$ show pre-operative photographs of a female patient with right-sided PRS who was received five fat grafting procedures from the age of 27 over a period of 3 years. Post-operative photos of the patient aged 31 taken 16 months after the final procedure show good symmetry (Figure 2a-b).

Autologous fat injections are a well-established plastic and aesthetic surgical technique that is used to reconstruct a variety of soft-tissue defects. It is minimally invasive and can be safely repeated over multiple sessions to gain precise soft-tissue augmentation and is superior to bulky flap-based reconstructions in maintaining natural facial contour and expression [2]. Fat resorption over time is the main limitation of this method and poorer fat retention rates were found in PRS patients when compared to patients receiving facial fat transfer for cosmetic or nonsyndromic reasons ( $40 \%$ vs $81 \%$ ) [3]. PRS was also identified as an independent negative predictor of fat retention, along with age, previous craniofacial bone surgery, grafted volume [4]. It is thought that the suboptimal blood supply in the recipient beds of PRS patients compromises fat graft take. This has led to many authors' preference toward overcorrection despite an increased risk of necrosis and cyst formation [2, 3]. Studies have observed an improvement in fat survival with subsequent procedures, attributed to establishment of vascular ingrowth in the recipient bed by the initial procedure, thus facilitating fat graft survival [4]. Despite the occurrence of fat resorption, overall patient satisfaction was high in our cohort and this is reflected in the literature where $87 \%$ of patients were satisfied with results [2]. Satisfaction levels were not limited to volume augmentation but could be credited to the improvement in facial symmetry, improvement in native skin and resolution of dark skin pigmentation [2, 4]. Other complications of fat transfer include fat necrosis, oedema and ecchymosis, and graft volumetric changes as was observed in 2 of our patients [5].

Patients were on average 37 years old in this study at time of receiving fat transfer, despite a majority with a much earlier presentation of disease. There is a general consensus that stabilisation or remission of disease should be achieved before starting treatment to avoid reversal of reconstructive efforts [3]. Some have suggested that fat grafting in the active state of the disease may inhibit or slow progression, and may even contribute in stimulating tissue regeneration [5].

In conclusion autologous fat grafting provides a successful method of soft tissue reconstruction with good aesthetic outcomes and high satisfaction rates for patients with hemifacial atrophy.

\section{Disclosure}

The authors declare no conflict of interest. 
Figure 1a, 1b: 27 year-old female patient presenting with right-sided hemifacial atrophy

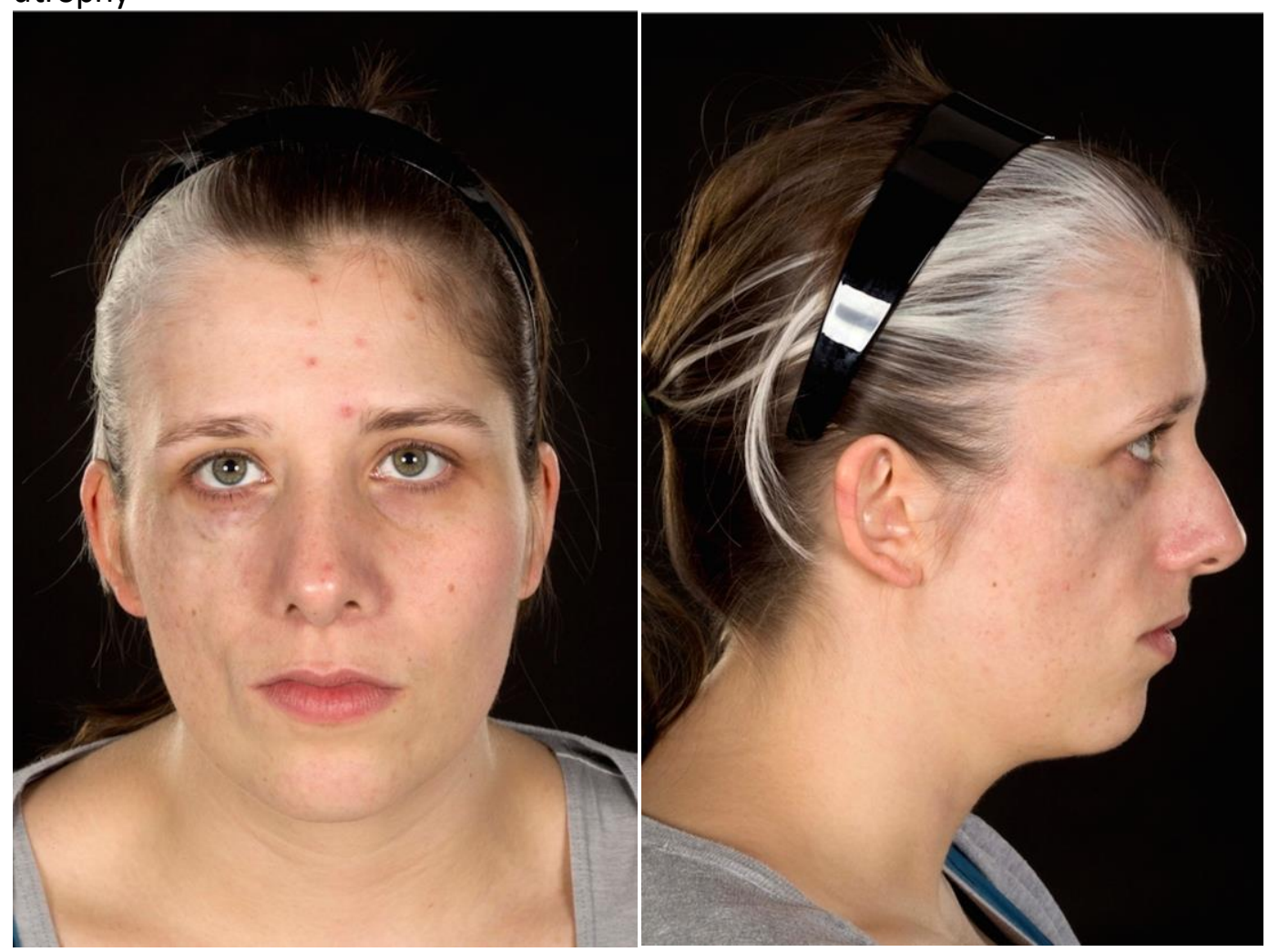

Figure 2a, 2b: 31 year-old female patient 16 months post-operative 5 fat transfer procedures to the right hemiface over a period of 3 years

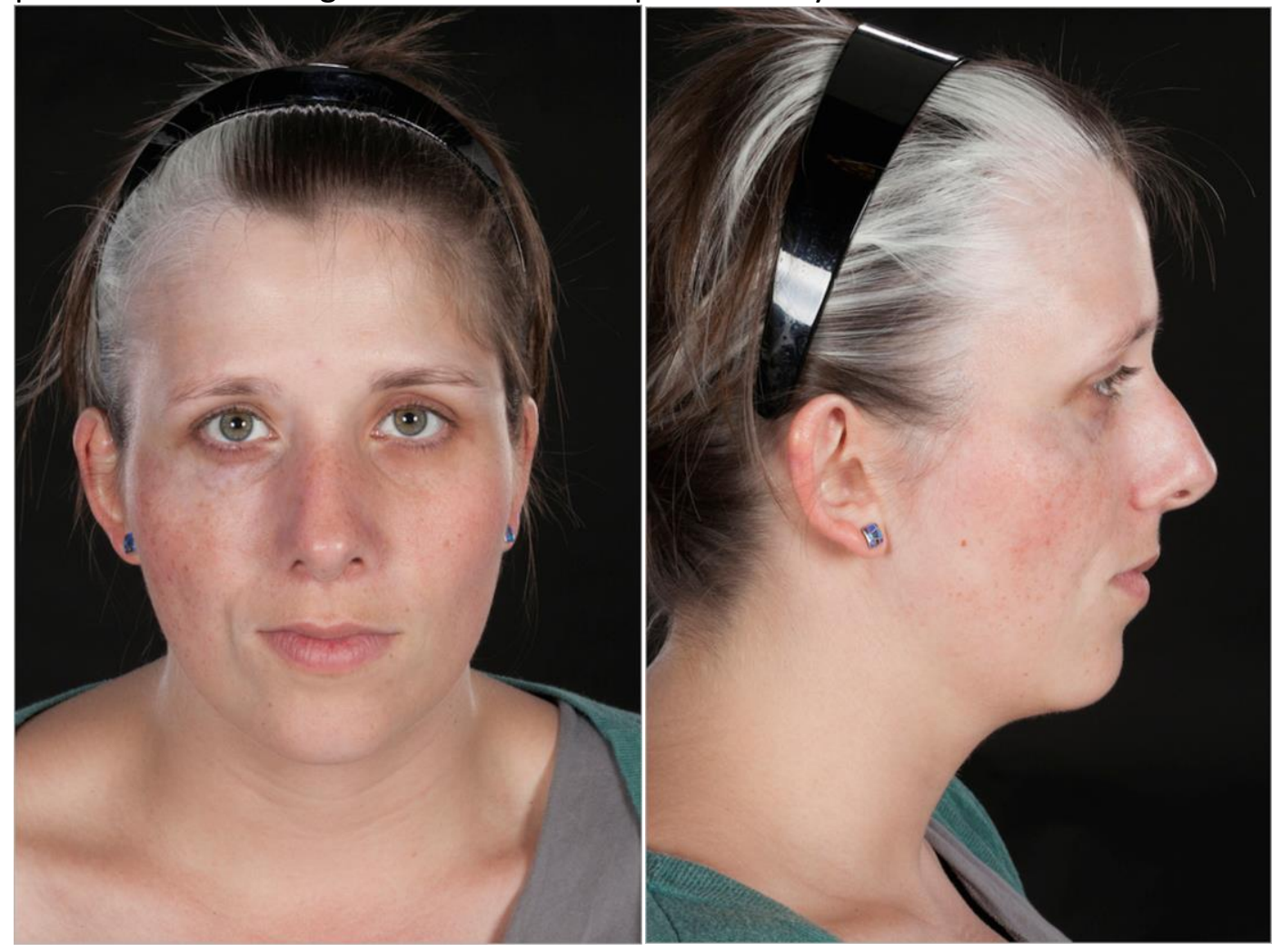




\section{References}

[1] Avelar RL, Göelzer JG, Azambuja FG, De Oliveira RB, De Oliveira MP, Pase PF. Use of autologous fat graft for correction of facial asymmetry stemming from ParryRomberg syndrome. Oral Surg Oral Med Oral Pathol Oral Radiol Endod.

2010;109(2):e20-e25.

[2] Rodby K, Kaptein Y, Roring J, Jacobs R, Kang V, Quinn K et al. Evaluating Autologous Lipofilling for Parry-Romberg Syndrome-Associated Defects: A Systematic Literature Review and Case Report. Cleft Palate Craniofac J. 2016;53(3):339-350.

[3] Slack, G. C., Tabit, C. J., Allam, K. A., Kawamoto, H. K., \& Bradley, J. P. (2014). Parry-Romberg Reconstruction: Beneficial Results Despite Poorer Fat Take. Annals of Plastic Surgery, 73(3), 307-310.

[4] Denadai R, Buzzo CL, Raposo-Amaral CA, Raposo-Amaral CE. Facial Contour Symmetry Outcomes after Site-Specific Facial Fat Compartment Augmentation with Fat Grafting in Facial Deformities. Plast Reconstr Surg. 2019;143(2):544-556.

[5] Hunstad JP, Shifrin DA, Kortesis BG. Successful Treatment of Parry-Romberg Syndrome With Autologous Fat Grafting: 14-Year Follow-up and Review. Ann Plast Surg. 2011;67(4):423-425. 